Les sources de l'Histoire des Mines : Nouveaux outils, Nouvelles approches

\title{
La mise en œuvre de la méthode de Renau d'Élissagaray
}

Renau d'Élissagaray's method at work

\section{Hélène Vérin}

\section{OpenEdition}

\section{Journals}

Édition électronique

URL : http://journals.openedition.org/dht/684

DOI : $10.4000 /$ dht.684

ISSN : 1775-4194

Éditeur :

Centre d'histoire des techniques et de l'environnement du Cnam (CDHTE-Cnam), Société des élèves du CDHTE-Cnam

\section{Édition imprimée}

Date de publication : 1 décembre 2008

Pagination : 187-197

ISBN : 978-2-95-30779-2-6

ISSN : 0417-8726

Référence électronique

Hélène Vérin, «La mise en œuvre de la méthode de Renau d'Élissagaray », Documents pour l'histoire des techniques [En ligne], 16 | $2^{\text {e }}$ semestre 2008, mis en ligne le 05 octobre 2010, consulté le 08 septembre 2020. URL : http://journals.openedition.org/dht/684; DOI : https://doi.org/10.4000/dht. 684

() Tous droits réservés 


\title{
La mise en œuvre de la méthode de Renau d'Élissagaray
}

\author{
Hélène Vérin \\ CNRS Centre A. Koyré
}

\begin{abstract}
RÉSUMÉ
La mise en œuvre de la méthode de Renau commença dans les ports dès 1680 . L'objectif se bornait à régulariser les différents tracés des carènes, propres aux principaux maîtres-charpentiers des ports. Le premier travail consista donc à la diffuser parmi eux, sous la conduite de Renau qui procédait luimême aux calculs nécessaires pour l'utilisation de son instrument, un ellipsographe. Par ailleurs, dès 1682, elle entrait dans le programme des écoles de construction des ports, destinées aux officiers. En 1684 on comptait trois frégates: I'Arrogant, l'Apollon et Le Brave construits selon ce nouveau procédé. En 1686, Tourville affirmait que les plans de maître Blaise Pangalo, l'un des meilleurs charpentiers du royaume, étaient faits selon cette méthode. À partir de 1692, sous Pontchartrain, l'application de la méthode de Renau entra dans une seconde phase : la détermination des proportions ellesmêmes et non plus simplement une régularisation des tracés. Les qualités nautiques remarquables de la frégate Le Bon en furent le couronnement.
\end{abstract}

Résumés et mots clés en anglais sont regroupés en fin de volume, accompagnés des mots clés français

a première mention que l'on ait retrouvée d'une Lmise en œuvre de la méthode de tracé de Renau date du 26 mai 1680. Le vice-amiral Tourville écrit de Rochefort à Seignelay pour rendre compte de l'épreuve qui en a été faite. Elle a consisté à faire tracer les gabarits d'un petit navire par le charpentier napolitain Blaise Pangalo' selon ses proportions et sa manière habituelle et de les faire tracer en même temps par Renau, à l'aide de son ellipsographe, sur les proportions de Blaise. L'épreuve consiste donc uniquement à vérifier si la méthode de Renau permet d'obtenir un tracé géométrisé qui corresponde bien avec celui que Blaise propose pour construire «le petit navire que nous faisons présentement ». Le résultat est un semiéchec, selon Tourville qui organise l'épreuve :

...Je n'ay pas manqué de faire examiner aux Mes charpentiers la machine du Sr Renauld et luy ay donné des proportions qu'il m'a demandé pour

1 Tourville avait ramené Blaise Pangalo de Tunis en 1679. Ses compétences et son intelligence du métier furent utilisées pour stimuler celles des charpentiers des ports. construire le petit navire que nous faisons presentement. On a trouvé qu'il n'avoit pas reussi et qu'il n'avoit pas tracé les gabarits qui conviennent a ce vaisseau avant de le faire commancer ${ }^{2}$.

Mais Tourville veut vérifier aussi la valeur du tracé de Blaise, et la soumet aux charpentiers et à Renau « qui conviennent qu'elle est bonne et seure ॥. II conclut sa lettre à Seignelay en l'assurant qu'il « ne desespere pas neantmoins qu'on ne puisse faire servir la machine du Sr Renauld en luy donnant des lignes convenables au vaisseau et avec lesquelles il puisse trouver quelques ouvertures pour tracer ensuite tous les gabarits ${ }^{3}{ }^{3}$. Tourville demande alors l'expertise de Feri, ingénieur du roi détaché à la marine à Rochefort :

J'ay faict observer au Sr Feri les lignes que font les acculemens et donné les diminutions des varangles du charpentier napolitain et du Sr Renault.

Il les a trouvé semblables avec cette seule diffe-

2 Archives nationales [ensuite AN] : Mar B/3/33 fo 412 , lettre de Tourville au ministre, Rochefort.

$3 \mathrm{lbid}$. 


\section{La mise en œuvre de la méthode de Renau d'Élissagaray}

rance que celle de Me Blaise estoit plus facile a faire comprendre aux charpentiers que celle du Sr Renauld (qui) ne se font que par un calcul difficile.

Nous avons une réponse plus précise de Feri où il explique qu'il $a$ :

examiné la machine du Sr Renau. Son grand gabarit est bon et convient avec celui du maîtrecharpentier; il a tiré les gabaris du fond avec sa machine ; mais il ne nous a point encore fait voir de demonstration juste pour ce qui regarde les gabaris de l'avant et de l'arrière, et il faut faire autant de gabaris de planche qu'il en a fait en bois, parce que sa machine ne peut opérer que dans un lieu uni et ne peut se porter sur une pièce de bois pour le gabarier ${ }^{4}$.

La remarque est d'importance : en effet, l'un des arguments de Renau est l'économie de bois que sa machine procure. En tout état de cause, il est inévitable qu'une innovation technique exige des ajustements collatéraux des pratiques qui, le plus souvent, n'apparaissent qu'au moment de la mise en œuvre.

Sur la demande du ministre, on est alors dans tous les arsenaux de marine en plein travail de relevé de mesures afin de régler les proportions des vaisseaux et le 5 juin Seignelay félicite Tourville d'entrer « dans un détail aussy utile que celuy-là ». II souligne l'importance que l'on attache à la Cour au perfectionnement de la construction navale: «si par suite de votre travail vous pouvez régler les proportions que doivent avoir les vaisseaux de S.M. et justifier cette connoissance par la bonté dont se trouvera le vaisseau que vous faites construire à Toulon, vous aurez l'honneur d'être parvenu à la plus importante chose qui puisse être entreprise dans la marine $\|^{5}$. Cette dernière incise, avec son appel à l'honneur - vertu noble par excellence - d'un grand officier de mer, et ceci pour son engagement dans des perfectionnements techniques, signale une inflexion remarquable des valeurs sociales de l'époque.

Seignelay semble mettre beaucoup d'espoirs dans la méthode de Renau. Le 22 juillet il demande à nouveau à Tourville de lui donner « son sentiment sur la machine dont le Sr Renau se sert pour tracer les gabaris des vaisseaux $1{ }^{6}{ }^{6}$. Les résultats de cet usage se sont

4 Lettre du 6 août 1680 citée par Jean Boudriot, Le vaisseau trois-ponts du chevalier Tourville, Paris, coll. Archéologie navale française, Ancre, 1998, p. 25.

5 BnF : n.a.f. ms 9481 p 249, lettre du ministre à Tourville. 6 AN : Mar B/2/43(2) fo 348, lettre de Seignelay à Tourville, Calais. améliorés, puisque Renau s'applique à « montrer aux charpentiers de Rochefort la manière dont ils doivent s'en servir "I, passant ainsi à une seconde étape de sa mise en œuvre dans les ports. Le 10 août il écrit à Seignelay pour l'en avertir, et celvi-ci lui recommande « d'en expliquer l'usage au fils de Me Hubac avant de venir icy $\|{ }^{7}$.

En octobre, Renau a quitté Rochefort pour la Cour, mais Seignelay continue son enquête dans les ports sur sa machine. Le 7 puis le 27 octobre 1680, il écrit à de Seuil, intendant de Brest, que «Sa Majesté veut qu'il fasse examiner par Hubac le pere la machine que son fils a veu a Rochefort et qui a esté inventée par le Sr Renaud et qu'il fasse sçavoir les sentimens dudit Hubac père ${ }^{8}{ }^{8}$. Au début de janvier 1681 Langeron, inspecteur de la marine, annonce l'arrivée de Renau à Brest, et celui-ci reprend son travail auprès des charpentiers du port, Hubac père et fils et de Salicon, charpentier du Havre qui se trouve dans l'arsenal. Renau semble réussir de mieux en mieux dans son effort de divulgation de sa méthode et tend à s'enthousiasmer dans sa correspondance avec Seignelay. Celui$c i$, dans une lettre du 16 juin 1681 lui fait part de sa satisfaction et précise quelques points importants qui délimitent ce que l'on attend de lui, c'est à dire trois choses : faire des relevés aussi précis que possible pour perfectionner ses tracés, apprendre sa méthode aux charpentiers mais encore, écrit Seignelay, « la principale chose à laquelle vous devez vous occuper est à travailler tousjours à perfectionner ce qui regarde la construction des vaisseaux et il he faut pas que vous vous persuadiez que vous soyez encore arrivé au point de cette perfection ». E† il réitère ses directives dans l'immédiat : "s surtout souvenez vous que ce n'est pas à vous de déterminer leurs proportions, mais à suivre avec votre machine celles qui sont déterminées par les maîtres charpentiers $\|$ ? .

D'après ce qu'écrit Seignelay, on peut admettre que Renau commençait à envisager de fixer luimême les formes à donner aux fonds. Ce qui lui est refusé et ce qu'il finira par obtenir bien plus tard, sous Pontchartrain. Pour l'heure, Renau demande à partir de Brest pour le Havre. Seignelay le lui accorde, mais seulement « après la construction des deux chaloupes que le Roy a ordonné estre bastie à Brest pour espreuve ॥. II s'agit d'un projet proposé par Tourville le 30 mai 1680 : faire construire par Hubac et Blaise deux

7 AN : B/2/43 fo 394, lettre de Seignelay à Renau (Rochefort). 8 AN : Mar B2 $42 f^{\circ} 331$, Ordinaire du Roi à de Seuil, Brest. 9 AN : Mar B/2/45 fo 227-228 et BnF naf ms 9481 p 276, lettre de Seignelay à Renau. 
modèles d'un navire de 66 pièces au petit pied « de même longueur et du même échantillon de bois ». La conduite des fonds de Hubac étant très différente de celle de Blaise, il sera alors possible, en les éprouvant ensemble, de juger lequel est le meilleur. Ou bien encore, on pourrait

envoyer icy le fils d'Hubac qui examinera la maniere de bastir de Me Blaise qui selon toutes les apparences doit estre bonne et en feroit son profit porveu que la vanité ne l'empeschat point de le suivre ce qui est extremement a craindre parmy nos Me charpentiers qui ne croyent pas qu'on leur puisse rien montrer quoyque jusqu'icy ils ayent faict des fautes considerables et desquelles ils n'ont encore su se corriger ${ }^{10}$.

Finalement, comme l'indique la lettre de Seignelay à Renau du 7 juillet 1681, on décide de construire à Brest les petits navires en chaloupe, c'est-à-dire sans les œuvres-mortes qui seraient inutiles à l'épreuve " ${ }^{11}$. Celle-ci a lieu en août 1681, et le 2 septembre Seignelay confirme qu'il en attend « de nouvelles lumières pour ce qui regarde les constructions ${ }^{12}$.

Notons que c'est en 1681 que l'examen du Mémoire sur la construction des vaisseaux de Renau est demandé par Seignelay à l'Académie des sciences, section de mathématiques. Quatre mentions en sont faites dans les procès-verbaux des séances. Le samedi 3 mai,

Mr. Blondel a rendu compte a la Compagnie de la commission qui luy avoit été donnée, et à $M$. Mariotte par Mgr le Marquis de Seignelay d'examiner l'invention de Mr. Renault pour une nouvelle construction des vaisseaux, qui se fait avec plus de facilité et toujours d'une même manière, laquelle invention a esté approuvée de la Compagnie ${ }^{13}$.

Les samedi 14 juin 1681 et 23 juillet où l'on récapitule les « Mémoire des observations et traitez Mathematiques ausquels on a travaillé dans l'Académie Royale des Sciences depuis le mois d'Aoust de l'année 1680 jusqu'au 15e de juin 1681 " ${ }^{14}$, « pour ce qui regarde les Mechaniques, ou les inventions qui peuvent estre de quelque utilité, Mr. Blondel a rendu compte a la Compagnie [comme ci-dessus]. Elle est fondée sur

10 AN : Mar B/3/33 fo 4l4, Rochefort, lettre de Tourville au ministre.

11 BnF : naf ms 9481 p 280, lettre de Seignelay à Renau.

$12 \mathrm{BnF}$ : naf ms 9481 p 281.

13 Académie des sciences : procès-verbal 1681, fo 99.

14 Académie des sciences : procès-verbal fo 11 et $170 \vee^{\circ}$. une section Conique, et c'est le fruit de la Geometrie speculative $)$. E† on reparle du mémoire dans les mêmes termes le jeudi 4 décembre 1681, «la Compagnie s'estant assemblée extraordinairement et estant avertie que Sa Majesté la vouloit honorer de sa presence $\|{ }^{15}$.

Au début de l'année 1682 Renau est à Dunkerque et le 7 février Desclouzeaux peut écrire à Seignelay

Le Sr Renau a achevé de conformer sa methode aux manieres et aux proportions du Me charpentier d'icy, il nous fait un modelle de touttes celles que ce Me charpentier a desiré de luy que nous avons examiné et trouvé tres juste, et il est convenu qu'il n'a rien a desirer de plus que ce qu'il a veu dans ce modelle, et je souhaitte avec passion que Mgr luy face bastir cette annee une fregatte de 40 pieces sur ce mesme modelle, il ne reste plus aud. Sr Renau qu'a achever de luy apprendre sa methode a quoy il va travailler, ce Me charpentier a perdu sa femme depuis cinq ou six jours apres avoir esté quinze a l'extremité ce qui fait qu'il n'a pas travaillé avec led. Renau autant qu'il auroit esté necessaire joint qu'il a esté obligé de travailler a mettre les galiottes sur les chantiers ${ }^{16}$.

En effet, on est alors dans les préparatifs de la guerre contre Alger pour lesquels Renau a proposé ses fameuses galiotes à bombes dont deux sont en construction déjà à Dunkerque et trois devront l'être au Havre. II tarde à quitter Dunkerque malgré les ordres de plus en plus pressants de Seignelay, et ce n'est que le 24 février que Desclouzeaux peut annoncer qu'il doit être arrivé au Havre et en même temps faire part à Seignelay du souhait du maître charpentier de Dunkerque de

bastir une fregatte sur le modelle en bois que j'ay envoyé a Mgr tant parce que cela le fortiffieroit dans les idées qu'il a prises de la machine de $\mathrm{Mr}$ Renau que pour luy oster de l'esprit la perte qu'il a faite de sa femme, sy l'on faisoit bastir cet esté au Havre, n'y ayant rien à faire en ce port, il pourroit aller y passer quelques jours pour se fortiffier danns la connoissance qu'il a prise de cette machine ${ }^{17}$.

Tout porte donc à penser que Renau a mis au point l'usage de sa machine et que, malgré les difficultés de calculs qu'elle exige, certains parmi les principaux maîtres-charpentiers des ports de Rochefort,

15 Académie des sciences : procès-verbal $1681, f^{\circ} 127 v^{\circ}$.

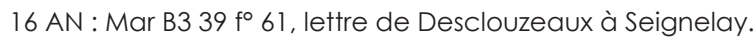
17 AN : Mar B3 39 fo 99, lettre de Desclouzeaux à Seignelay. 


\section{La mise en œuvre de la méthode de Renau d'Élissagaray}

Brest, le Havre et Dunkerque savent la mettre en pratique et s'efforcent de s'y perfectionner. Sans doute nul n'ignore le soutien que Seignelay - et au-dessus de lui, le roi - apporte à cette technique, mais on peut aussi penser que l'on était soucieux d'obtenir des tracés mieux contrôlés. Rappelons l'avantage, souligné à l'Académie des sciences, d'une « construction des vaisseaux qui se fait toujours d'une mesme maniere ". En tous cas l'intérêt de cette méthode est suffisamment reconnu pour que son enseignement soit proposé dans les écoles de construction des ports, destinés aux futurs officiers de mer. L'établissement, le développement et la définition des programmes d'enseignement de ces écoles est en effet à l'ordre du jour. Le Danois, commissaire de la marine au Havre décrit le 4 mai 1682, en réponse à l'enquête de la Cour sur le sujet, ce qu'enseigne au Havre «le Me charpentier pour l'escole de la construction »:

La première leçon est d'apprendre aux dits officiers le nom de chacune des pièces de bois employées à la construction d'un vaisseau, la manière qu'il faut les asssembler les unes avec les autres, l'endroit où elles se placent sur ledit vaisseau.

Quand ils auront pris une certaine connoissance de cette leçon, il doit leur montrer l'usage de la machine de M. Reynaut pour leur apprendre ensuitte à tracer et faire des plans de vaisseaux, et frégates, pour cet effet il demande à Mgr qu'il luy soit permis de faire un petit vaisseau dans le lieu de l'escole affin de leur pouvoir monstrer toutes les pièces qui donnent liaison.

Depuis un temps l'escolle a esté irrégulière parce que le Me charpentier s'est trouvé pressé par le travail de l'Arrogant et de trois galiottes. Comme il doit mettre dans peu le Brave sur le chantier, lesdits officiers pourront s'appliquer de même qu'ils ont fait jusqu'icy à voir tracer et faire tous les gabaris de chaque pièce et estre present lorsqu'on le monte ensemble ${ }^{18}$.

Ce dernier paragraphe rend compte de l'influence qu'a pris Renau dans la construction navale, puisque les galiotes sont de son invention, et que les deux autres bâtiments : l'Arrogant et le Brave sont tracés selon sa méthode et à l'aide de son ellipsographe.

En 1682 et 1683, Renau est très occupé par les préparatifs et le déroulement des expéditions en Méditerranée. Néanmoins, il continue à réfléchir aux conditions de l'application de sa méthode et en février 1684, répondant aux ordres de Seignelay qui lui demande de revenir à la construction navale, il lui envoie un Mé-

18 AN : Mar G/86, fo 12-15. moire sur les constructions ${ }^{19}$ où il expose comment il conçoit le rôle qu'il pourrait jouer dans le perfectionnement ef l'économie de la construction des vaisseaux du roi. Renau a acquis une autorité qui se ressent aussi bien dans la façon de présenter ses propositions, que dans leur teneur et au total, on le voit s'appuyer sur le bilan de son action dans la construction pour proposer le programme qu'il juge bon d'établir, à partir des résultats obtenus. II s'agit en effet ni plus ni moins dans ce mémoire, que des fonctions dont Renau «se prescrit ॥ comme il le conclut lui-même. Cela revient à institutionnaliser celles qu'il a remplies auprès des ports et des principaux maîtres-charpentiers et ainsi à généraliser l'usage de sa méthode. Ses arguments sont de trois ordres : sa méthode « s'est trouvée assez universelle, pour satisfaire généralement à toutes les manières de bastir des charpentiers de tous les ports ॥, elle a reçu l'appui de "Mrs de l'Académie », qui on reconnu son avantage, soit « que lorsqu'on parviendra à quelque vaisseau sans deffaut ou dont on sera entièrement satisfait on en puisse refaire tant que I'on voudra de pareils ॥. Enfin, elle a fait ses preuves: "l'Apollon et l'Arrogant qui se sont trouvés les meilleurs vaisseaux de l'escadre du Nord ont esté faits par cette méthode. J'ay aussi, ajoute-t-il, tracé le Brave au Havre de Grâce qui est un vaisseau qui n'a point encore servy duquel j' ay encore meilleure oppinion pour la finesse de la voillure $॥{ }^{20}$.

Renau expose alors le détail de ses fonctions : être présent à la construction de tous les vaisseaux; les faire tracer lui-même et

faire monter sur la quille le nombre de coupes necessaires en les nivelant et faisant $\left(22 v^{\circ}\right)$ observer les mesures avec la dernière précision pour pouvoir s'assurer d'une grande justesse tant dans le tracement du plan, que pour la conformité qu'il faut qu'il y ait entre la figure que ces coupes feront avoir au vaisseau et celle du plan "), un autre effet de ces dispositions étant d'accoûtumer les charpentiers à de grandes précisions de mesures qui sont absolument nécessaires.

L'objectif est de se donner les moyens de reproduire exactement un bâtiment construit, faire pour chacun d'eux « un plan accompagné d'un mémoire, qui contienne toutes les mesures et tous les points qui prescrivent et déterminent toute la fabrique $»$.

Vient alors le contrôle des qualités/défauts à la mer : pour mettre en place les conditions d'un suivi rigoureux, Renau définit les critères essentiels de mar-

19 AN : Mar D/1/10 fo 22-25.

$20 \mathrm{ld}$., f० 22. 
che dans différentes conditions dont il devra être tenu informé de sorte que « sçachant d'ailleurs les différantes figures, on découvre autant que l'on pourra par les règles des forces mouvantes, les causes de ces différans effets, et en suitte la figure la plus convenable pour la plus grande perfection $॥$.

Il est intéressant de noter que Renau considère que le recours à la connaissance des « forces mouvantes $"$ ne peut servir le projet de perfectionnement et de contrôle des effets que si l'on se donne en outre le moyen de véritablement procéder à une comparaison, ce qui suppose aussi que l'on puisse croiser les informations, multiplier les sources de connaissance. Cette organisation aurait pour effet de modifier les conditions de l'apprentissage artisanal : les charpentiers lorsqu'ils seront contraints à plus de précision dans leur mesures, pourront non seulement reproduire strictement ceux qui se seront révélés bons, mais encore "lorsqu'une personne capable (en l'occurrence Renau) sera employée de la manière que je viens de dire tous les charpentiers tireront les mesmes lumières $\left(24 r^{\circ}\right)$ que si chacun avoit basty tous les vaisseaux que l'on fera qui est assseurément le seul moyen de réussir $\%$. Le décloisonnement des savoir-faire artisanaux par la diffusion de méthodes réglées mathématiquement est l'un des thèmes majeurs de la réduction en art des pratiques, à laquelle le mémoire de Renau contribue, dans le champ de la construction navale. Or ce décloisonnement avait donné lieu à la mise en place de conseils réunissant des officiers de terre et de mer, des charpentiers des ingénieurs du roi en fonction dans le port... L'idée de recourir à la confrontation réglée d'opinions et de points de vue divers, pour le perfectionnement des projets et selon une approche tout empirique est raillée par Renau qui appuie ses arguments en faveur de son système, par une critique impitoyable de ces conseils de construction qui allient, selon lui, la confusion de disputes mal étayées, à l'incompétence scientifique de ses membres. Sans doute s'avance-t-il beaucoup lorsqu'il se permet une critique aussi radicale d'une institution voulue par Colbert, même s'il ne manque pas d'y associer des raisons d'économie en matière première et en main d'œuvre. En tous cas, il n'obtient pas gain de cause. Rappelé par Vauban alors engagé dans la guerre de Flandres, il participe au siège de Luxembourg ${ }^{21}$, puis retourne auprès de Seignelay qui dirige la campagne contre les Génois et ensuite contribue au siège de Cadequier en juin 1684. Après quoi il travaille à Toulon avec Blaise Pangalo sur différentes machines.

21 Du 28 avril au 3 juin 1684.
Tourville qui est à Toulon écrit en novembre 1685 à Seignelay:

Quoyque vous ne me parliez plus de construction cela n'empesche pas que je n'y ayt plus d'aplication que je n'ay et jay aprit de Renault a trasser toutes les lignes qui peuvent convenir a un navire on les trasse avec la machine qui est d'une plus grande estendue que le compas mais pour s'en servir il faut beaucoup plus de mesures que les charpentiers n'en donnoient, j'espere quelque jour que vous aurez assez de confiance en moy pour me donner la conduite de la construction d'un vaisseau'22.

Durant cet hiver 1685-1686, Renau, Pangalo et encore Tourville travaillent ensemble à Toulon et le 19 janvier 1686, Renau quittant le port pour la Cour, Tourville lui confie une lettre pour Seignelay où il fait état de cette collaboration et de ses résultats, qu'il juge très satisfaisants pour la marine du roi :

Je vous envoye les esperons des vaisseaux de chaque rang par Monsieur Renauld qui s'en est bien voulu charger. II vous expliquera toutes les raisons que je luy ay dittes afin de vous faire cognoistre de la maniere qu'ils sont construits et proportionnes. Il pourra dire aussy comme j'ay continuellement travaillé avec luy sur les constructions et j'ose vous assurer que je n'ignore en aucune manière la methode de M. Renault qui est sans contredit la meilleure de toutes et que j'ay apris a fonds, j'ay remarque que les plans de Me Blaise sont faicts sur cette methode qu'il a parfaitement bien comprise et dont il se sert bien. Je vous avouerai mesme que ce charpentier ayant un genie merveilleux conceut dans un instant la methode de M. Renault et dict que chacun travaillois à sa maniere cependant il est constamment vray que c'est a luy qu'on a l'obligation de toutes les regles qu'on a dans la marine. Mais aussy il avoit affaire a un homme penetrant comme M. Renault vous avouera luy mesme, qui n'a jamais cognu un homme qui en eu davantage. Et je vous diray que tout ce que M. de Langeron peut sçavoir ne sont tirés que de cette methode et qu'on ne fera jamais si bien que de s'en servir totalement de la maniere que je l'ay approfondie. II vous entretien-

22 AN : Mar B4 $10 f^{\circ} 167-170 v^{\circ}$, lettre de Tourville à Seignelay $169 v^{\circ}$, Toulon le 23 novembre 1685. 


\section{La mise en œuvre de la méthode de Renau d'Élissagaray}

dra aussy Mgr de plusieurs autres choses dont je l'ay charge ${ }^{23}$.

Ainsi, en 1686 la méthode de tracé de Renau, combinée à la « manière » de Blaise Pangalo aurait abouti à une maitrise des façons que Tourville juge très satisfaisante.

Entre 1686 et 1691, Renau est appelé à d'autres tâches que la construction navale. II prépare et publie anonymement et avec l'aide de Joseph Sauveur, sa Théorie de la manœuvre des vaisseaux et le 28 janvier 1689 il est nommé par Louis XIV académicien honoraire, premier titulaire. II est auprès de Vauban durant la campagne de 1688 aux sièges de Philisbourg ${ }^{24}$, où il est blessé, de Frankenthal et de Manheim ${ }^{25}$ où il se distingue. En 1689, alors qu'il est encore sous les ordres de Vauban, il est rappelé par Seignelay et embarque pour une campagne de mer et lorsque le 23 juin 1690 la flotte quitte Brest pour la campagne navale contre les anglo-hollandais, Renau est auprès de Tourville sur le Soleil Royal.

Ce n'est qu'en 1691 qu'il va enfin pouvoir réaliser un petit bâtiment, sur des proportions qu'il a lui-même fixées. En novembre 1690 Seignelay a disparu et c'est le comte Pierre Louis Phélipeaux de Pontchartrain qui le remplace. On a souvent affirmé qu'il n'appréciait pas Renau. Sans doute est-ce à la volonté du roi que ce dernier doit sa promotion comme officier de la marine royale, que Seignelay lui avait semble-t-il promise. Mais encore, si l'on suit la correspondance de Pontchartrain et Renau on s'aperçoit que celuici a gagné la confiance du ministre qui plus tard, lui confiera même son fils Jérôme pour son instruction.

C'est donc durant l'été 1691 que le roi ordonne " au Sr Renau de faire bastir un yack au retour de l'armée navalle sur les proportions qu'il doit donner ». Pontchartrain précise à Desclouzeaux qu'« il est necessaire que vous luy fassiez fournir les ouvriers et les matieres necessaires pour cette construction et que vous luy donniez toutes facilitez dont il aura besoin pour la faire achever promptement $"{ }^{26}$. Mais Renau, aux prises avec les officiers de Brest qui refusent de suivre les cours de manœuvre dont le roi lui a donné charge, tombe malade ${ }^{27}$. Le roi le rappelle à la cour

23 BnF : naf ms 9481 p 296, lettre de Tourville au ministre, TouIon le 19 janvier 1686.

24 Du 29 septembre au ler novembre 1688.

25 Respectivement les 6-12 et 17-19 novembre 1688.

$26 \mathrm{AN}$ : Mar $\mathrm{B} / 2 / 79$ fo 311 , lettre de Pontchartrain à Desclouzeaux, Versailles le 21 août 1691.

$27 \mathrm{AN}$ : Mar $\mathrm{B} / 3 / 52 \mathrm{f}^{\circ} 3-4 \mathrm{v}^{\circ}$, lettre de Renau à Pontchartrain, par une lettre du 16 septembre et ce n'est que le 19 octobre que Pontchartrain envoie une nouvelle lettre à Desclouzeaux pour lui annoncer la décision de construire ce yack au Havre

"Le Roy a ordonné au Sr Renau de se rendre incessamment au Havre de grace pour bastir un yack suivant une nouvelle metode qu'il pretend avoir. L'intention de Sa Majesté est que vous luy fassiez fournir les ouvriers et les matieres necessaires pour faire ce yack $\|{ }^{28}$.

Bien sûr cette construction n'est pas prioritaire, et Pontchartrain précise à Louvigny qu'

" a l'esgard du yack que le Sr Renaud doit bastir je vous ay fait sçavoir qu'il ne devoit estre construit quen cas qu'il ne causa aucun retardement a ce vaisseau [Le Juste alors en construction au Havre] $11{ }^{29}$.

Au mois de décembre, la construction du petit bâtiment est lancée. Selon Pontchartrain,

« Sa Majesté est bien aise d'apprendre vostre arrivée au Havre, je ne doute point que vous n'ayiez su mettre sur les chantiers le petit bastiment que vous devez faire faire, il est important que vous pressiez la construction affin de sçavoir au plus tost a quoy vous tenir, sur cela escrivez moy touttes les semaines les diligences que vous faites affin que j'en puisse rendre compte au Roy $1{ }^{30}$.

Le 15 , il demande

un dessein affin que j'en puisse rendre compte a S.M. et que je puisse luy faire voir par ce dessein la difference qu'il y a de la metode ordinaire a celle dont vous pretendez vous servir. Vous pourrez revenir icy lorsque ce bastiment sera en estat de ne pouvoir recevoir aucun changement, [...] ainsy pressez le travail de maniere que ce bastiment puisse estre dans peu en cet estat apres quoy il n'y aura point d'inconvenient que vous veniez icy ${ }^{31}$.

Brest le 10 septembre 1691 : « depuis dix ou douze jours j'ay esté arresté au lit par une fluction sur les yeux et à la teste qui m'a empesché d'avoir l'honneur de vous envoyer tout ce que vous m'avee ordonné et de travailler à préparer ce qui est necessaire pour bastir le petit yackj'espere estre en estat ॥. 28 AN : Mar B/2/80 fo 688, lettre de Pontchartrain à de Louvigny (Le Havre) du 19 octobre 1691.

$29 \mathrm{AN}$ : Mar $\mathrm{B} / 2 / 80 \mathrm{f}^{\circ} 734$, lettre de Pontchartrain à de Louvigny (Le Havre) du 23 octobre 1691.

$30 \mathrm{AN}$ : Mar B/2/80 fo $1026 \mathrm{v}^{\circ}-1027$, lettre de Pontchartrain à Renau (au Havre), Versailles le 4 décembre 1691.

31 AN : Mar $B / 2 / 80 f^{\circ} 1122 v^{\circ}-1123$, lettre de Pontchartrain à Renau (au Havre), Versailles le 15 décembre 1691. 
Le petit bâtiment est une frégate légère de 16 canons, la Serpente. En mars 1692, l'épreuve doit en être faite au Havre et le 1er avril Renau peut écrire à son ministre qu'il a fait éprouver les qualités nautiques de la Serpente en la faisant naviguer en même temps qu'un capre qui a la réputation d'être parfait.

Mgr je puis tousjours vous asseurer qu'au moins le petit bastiment que je vous ay fait faire vaudra beaucoup plus que beaucoup d'autres que le Roy a. Avant de prendre aucune resolution sur cette construction il seroit bon que j'eusse l'honneur de vous entretenir un quart d'heure avec un modele en bois que jay fait faire pour pouvoir vous expliquer certaines choses qui seront de consequence. Si vous n'avez pas besoin de moy davantage icy Mgr, je pourrois vous aller rendre compte de cette construction incessamment.

La petite fregate est restee dehors si le temps le permet j'iray encore faire un essay tantost, parce que j'ay encore fait changer quelque petite chose aux voiles ${ }^{32}$.

Des épreuves sont organisées qui donnent lieu à trois rapports qui nous sont parvenus et signalent un bon comportement de la Serpente : par toutes sortes de temps, « on a esprouve ses qualites avec toutes les meilleures valeurs d'issy. Je crois monsieur d'après cela que vous devez estre content soyez sur que si s'estoy mon ouvrage propre je n'en auray pas plus de joye $"{ }^{33}$. Aussitôt Renau envisage de faire construire une autre frégate et propose quelques modifications

Si je m'assure de ne pas faire tirer plus d'eau au vaisseau derrière que devant ce qui seroit d'un tres grand advantage en ce que sans plus faire tirer tant d'eau que les vaisseaux ordinaires, je leur donnerois cependant plus de creux et les ferois par là d'un plus grand port, comme la petite fregate que j'ay faite gouverne par excellence et que ce tirage d'eau plus derriere que devant aux vaisseaux de mer n'est que pour les faire gouverner il me paroist que sans ce secours par ma construction ils ne laisseroient pas que de gouverner parfaitement bien par la construction du bastiment que je demande a faire je m'en assureray aussy bien que de plusieurs autres choses qui tendent a la perfection si vous donnez vos ordres a Brest Mgr ce petit bastiment pourroit estre esprouve dans l'annee avant son retour, n'estant pas necessaire de plus de six semaines de temps pour

32 BnF : naf ms 9481 p 319 : Le Havre, Lettre de Renau au ministre Pontchartrain.

33 Id., p. 31, rapport de Marquaysac, le Havre, 20 avril 1692. le construire, apres quoy, Mgr, nous pourrions agir seurement et vous faire les meilleurs bastiments de la mer Je renvoye pour cet effet incessamment le Sr Brun a Brest ${ }^{34}$.

Pontchartrain accède à cette dernière demande de Renau puisque le 28 mai il rappelle : « Je vous ay desja donné advis que j'avois ordonné a Brest qu'on donnat au jeune Brun tout ce quil luy faudra pour la fregate qu'il y doit bastir suivant vostre methode ${ }^{3}{ }^{35}$. Renau est à la Hougue, et six jours avant le fameux désastre du 2 juin, il manifeste son inquiétude devant les faiblesses de la flotte française, reprend aussi son idée d'un remaniement des carènes et le problème du tirant d'eau : « II faut faire asseurement bastir promptement le plus grand nombre de gros vaisseaux qu'il se pourra si le Roy est en estat de le faire...et si le Roy prend ce party la, Mgr, il faut un peu conferer sur la nature de ces vaisseaux. II faut en faire un nombre de cent canons c'est a dire de 80 en trois batteries. II ne faudra pas qu'ils tirent tant d'eau que les nostres. II faudra aussy conferer sur beaucoup d'autres choses $1{ }^{36}$.

La construction de la nouvelle frégate de Renau par le fils Brun est avancée et le 6 août 1692, Pontchartrain écrit à Desclouzeaux, intendant de l'arsenal : « Je vous ay envoyé les ordres pour l'armement de la fregatte construite sur les proportions du Sr Renau, il est necessaire que vous laissiez au jeune Brun qui la construit, la liberté de faire les mâts et les voilles de la maniere qu'il le jugera a propos $\|^{37}$. Le 16, il écrit à Renau : « J'ay donné les ordres necessaires, pour l'armement de la fregate qui a esté bastie à Bastie (Brest), suivant vostre methode, vous avez bien fait d'informer le Sr Trullet de ce qu'il y a a observer dans son armement $1{ }^{38}$.

Je n'ai rien retrouvé sur l'essai de cette frégate dont l'armement a peut-être été retardé : on craint une descente des Anglais, et Renau, jusqu'au printemps suivant, est très occupé par la fortification des côtes de nord Bretagne et de questions de guerre navale. En mai 1693 il est chargé de l'essai de deux frégates, la Mutine et l'Héroïne. Selon les capitaines qui

34 ld., p. 313, lettre de Renau au ministre.

35 AN : B2 84 f॰657, lettre de Pontchartrain à Renau (La Hougue), Paris le 28 may 1692.

36 BnF : n.a.f. ms 9481 p 315, lettre de Renau au ministre.

37 AN : Mar B2 $85 f^{\circ} 272$, lettre de Pontchartrain à Desclouzeaux du 6 août 1692.

38 AN : Mar B2 85 fo 379, Antoine Trullet, lieutenant de vaisseau à Brest depuis 1679, nommé capitaine en 1692. II commandait les batteries du Soleil Royal à la victoire de Beveziers. 


\section{La mise en œuvre de la méthode de Renau d'Élissagaray}

les ont fait passer de Brest à St Malo, «il s'en faut beaucoup qu'elle (l'Héroïne) ne marche aussi bien que la Mutine ». " II est bien important », ajoute Pontchartrain pour Renau, « avant de commencer ce vaisseau de 60 pieces, que vous examiniez avec soin les deffauts de cette fregate, afin de les corriger ${ }^{39}$. Renau écrit à Pontchartrain pour lui faire part de ses propres remarques sur l'Héroïne, mais ce semi-échec ne remet pas en cause le projet en cours, soit « les ordres que le Roy a donné pour la construction d'une fregate de 50 a 54 pieces de canon a Brest sur les mesmes proportions $1{ }^{40}$ et le 17 juin Pontchartrain peut écrire à Renau :

Je suis bien aise d'apprendre que vous avez achevé de tracer le plan de la fregate de 50 canons que vous devez faire bastir a Brest aussy tost qu'elle sera en estat de pouvoir estre achevée par un contremaître sans qu'il soit a craindre que l'on gaste rien a sa construction j'envoyeray le jeune Brun au Havre pour mettre sur les chantiers celles qui y doivent estre basties faites moy scavoir quand vous estimez que ce charpentier pourra partir de Brest ${ }^{41}$.

Renau répond:

La fregate que nous avons mise sur le chantier a toutes ses couples en place, on l'achevera de lisser du haut en bas demain apres quoy on avancera le reste extremement viste de maniere que le Sr Brun pourra partir pour le Havre dans trois semaines ou environ, il ne peut guere auparavant parce qu'il est bon qu'il conduise les ponts, les ceinstres et un peu les oeuvres mortes a cause que la tonture de toutes ces choses est autrement que dans la construction ordinaire ayant des relevemens differens et d'autres proportions ${ }^{42}$.

Le 24 juillet Renau annonce que la fin de la construction est prévue pour le 17 août:

Je suis encore resté icy pour examiner plus exactement le detail de la construction de la fregatte que l'on y fait sur ma methode afin que si cette construction reussit de voir si on ne pouroit pas establir un ordre a l'avenir pour espargner considerablement en journées d'ouvriers sur la construction de chaque vaisseau. Si vous ne me destiniez a rien Mgr je vous en demanderois le comman-

39 AN : Mar B2 $90 f^{\circ} 471-471 v^{\circ}$, lettre de Pontchartrain à Renau, 20 mai 1693.

40 AN : Mar B2 $90 f^{\circ} 574 v^{\circ}$, lettre de Pontchartrain à Renau, 30 mai 1693.

41 AN : Mar B2 90 fo $733 v^{\circ}$, lettre de Pontchartrain à Renau, 17 juin 1693.

$42 \mathrm{AN}$ : Mar D/1/10 ( $f^{\circ} 64$ ), lettre de Renau au ministre, Brest. dement pour 2 mois, ce seroit assez de temps pour remarquer ce qu'il y auroit a corriger, et on l'esprouveroit ainsi avec les bons voiliers de l'escadre ${ }^{43}$.

II s'agit du vaisseau Le Bon, «long de 132 pieds, large de 33 pieds 8 pouces, avec un creux de 14 pieds, ce vaisseau de 750 tonneaux de port en lourd était armé lors de son lancement de 56 canons et avait un équipage de 7 ou 8 officiers et de 330 matelots $"{ }^{44}$. Renau obtient le commandement de la frégate armée et le 3 février 1694 il devrait l'avoir pris comme l'écrit Pontchartrain à Desclouzeaux :

J'ay esté surpris d'apprendre que $M$. Renau ne fust pas encore arrivé a Brest le 25 je ne doute pas qu'il ne le soit a present et qu'il ne soit mesme prest a partir il est bon que le petit Brun qui a construit le vaisseau que le Sr Renau monte s'embarque pour observer pendant sa navigation ses bonnes et mauvaises qualites. Je luy donne ordre a St Malo où vous l'avez envoyé de repasser incessamment a Brest pour s'embarquer sur ce navire. L'autre charpentier que vous avez envoyé avec luy prendra soin de la construction des chaloupes. Je vous envoyeray incessamment la route pour les trois escouades de la compagnie de Fontenay qui doivent servir sur Le Bon ${ }^{45}$.

Renau doit croiser « à l' ouvert de la Manche ». Pontchartrain l'avise des décisions du roi, et en particulier :

Je donne ordre au petit Brun que M. Desclouzeaux a envoyé a St Malo, de retourner incessamment à Brest et de s'embarquer avec vous pour faire les observations que vous luy ordonnerez sur les qualités du vaisseau Le Bon. Je n'ay rien a vous prescrire sur cela, ne doutant pas que vous les examinerez de bonne foy, et que vous ne me fassiez scavoir a vostre retour s'il convient au service du Roy de se servir ou non de la nouvelle methode de construire que vous avez imaginé ${ }^{46}$.

Renau est chargé de remettre une lettre à Relingue qui doit contribuer à l'essai du Le Bon

Sa Majesté trouve bon que vous gardiez avec vous les vaisseaux Le Fort et Le Bon que vous trou-

43 AN : Mar D/1/10 - (f० 65-66), lettre de Renau au ministre, 24 juillet 1693, Brest.

44 Alain Demerliac, La marine de Lovis XIV, Nice, Oméga, 1992. Le Bon fut rayé des listes de la marine royale en 1702 OU 1703.

45 AN : Mar B2 97 f $^{\circ} 260 v^{\circ}-261$, lettre de Pontchartrain à Desclouzeaux du 3 février 1694.

$46 \mathrm{AN}$ : Mar B2 $97 f^{\circ}$ 263, lettre de Pontchartrain à Renau du 3 février 1693. 
verez dans ce parage ; et comme elle a fait armer ce dernier qui a esté construit sur une nouvelle methode pour en faire l'essay, et voir s'il conviendra a son service de se servir a l'advenir de cette construction ou non, son intention est que vous le fassiez éprouver contre tous ceux qui composent votre escadre, lesquels estant les meilleurs que le Roy ayt on jugera par la comparaison qu'on fera d'eux avec ledit Vaisseau Le Bon quel fonds on peut faire sur cette nouvelle methode ${ }^{47}$.

Le 8 février, Renau écrit à Pontchartrain tout ce qu'il espère du Bon et la réponse du ministre manifeste sa confiance :

J'ay receu la lettre que vous m'avez escrite le 8 de ce mois. Jay veu ce que vous me marquez a propos de la construction du vaisseau Le Bon. Je suis bien persuadé que vous m'en rendrez un compte fidele, et que vous n'aurez aucune prevision sur cela. Je vous prie pendant que vous serez a la mer d'avoir une attention particuliere a observer les bonnes et mauvaises qualites de cette construction parce que sur ce que vous m'escrirez, le Roy se determinera a faire faire d'autre examen ${ }^{48}$.

Renau doit quitter la croisière pour se rendre au parage de Belle-île et de Groye où I'on craint une attaque de corsaires sur des vaisseaux de retour des Îles de l'Amérique et c'est de la rade de Groye que Renau envoie son compte-rendu sur le comportement du Bon :

J'ay attendu que j'eusse un peu navigué sur le vaisseau Le Bon pour avoir l'honneur de vous informer comment il se comporte à la mer nous ne pouvions pas trouver un temps plus propre que celuy que nous venons d'avoir pour en faire une bonne espreuve, nous avons essuyé un coup de vent fort pesant de cinq jours il commença par le sud ovest forcé pendant six heures ensuite il mit de mesme a l'ouest, au nord ouest, et puis il sauta au nord est au sud est et revint au sud ouest et tousjours ventant a tout rompre. La mer estoit tres grosse, de manière qu'a tous ces changemens de

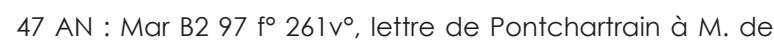
Relingue du 3 février 1694.

$48 \mathrm{AN}$ : Mar B2 $97 f^{\circ} 353 v^{\circ}$, lettre de Pontchartrain à Renau du 17 février 1694. Eł le 20 février « Je suis bien aise d'apprendre qu'il y a lieu de bien esperer de la construction du vaisseau Le Bon. Je vous recommande de toujours observer les bonnes et mauvaises qualitez avec toute l'exactitude possible afin de perfectionner cette maniere de construire $"$, id, fo $374 \vee^{\circ}$. vent nous l'avons eue tantost de bout, de costé et tantost derriere et agitee de toute part a tout cela Mgr nostre vaisseau n'a point tourmenté ny reçu dedans une goutte d'eau il ne tangue ny ne roule que tres peu, il capeye en perfection et court de l'avant, pour les commoditez de la mer il ne se peut pas voir un meilleur vaisseau il ne s'agit plus que de sçavoir s'il est fin de voile, ce qu'on ne peut bien juger qu'en se meuvant contre quelque bon voilier reconnu, nous n'avons encore eu aucune occasion de nous essayer veritablement nous avons seulement pris une caiche ${ }^{49}$ angloise de peu d'importance vent arrière les anglois disent cependant qu'elle va parfaitement bien a cette voillure. Nous portons fort bien la voille et nous gouvernons en perfection par toutes sortes de temps et a toutes voillures. Voila tout ce que je connois jusqu'a present de cette construction de laquelle j'ay tousjours de plus en plus meilleure opinion J'auray l'honneur de vous informer dans la suite de ce que j'en apprendray de nouveau ${ }^{50}$.

C'est ici que se situe un épisode mieux connu, parce qu'il donna lieu à une publication, la lettre du marquis de Nesmond, officier à bord du Le Bon, lettre publiée sous le titre de «Relation curieuse d'un combat naval donné par un vaisseau du Roy contre un Anglois, dont la perte est de plus de dix millions $1{ }^{51}$. La lettre commence par un panégyrique du Bon :

Me voici de retour à Brest, Monsieur. Le vaisseau du Roy le Bon s'est trouvé admirable ; il porte parfaitement bien la voyle; il va fort bien au plus près du vent; il tangue, \& roule si peu, que nous n'avons presque senti le gros tems qu'il a fait ; \& jamais Vaisseau ni Chaloupe ne gouverna mieux : en un mot, c'est le meilleur vaisseau qui ait parû : \& je ne doute point qu'on ne bâtisse desormais sur ce modèle, dont $M$. Renau est l'auteur.

De toutes les épr(e)uves que le Bon a souffertes, la plus rude est la dernière, sans contredit, \& voici comme elle se passa.

Suit la narration de la chasse, de l'abordage, du combat et de la reddition du navire, des richesses qu'il contenait, de la tempête et aussi de la conduite des hommes et en particulier de Renau.

" Jamais homme ne fit voir tant de sang froid qu'il en a fait paroître durant tout le combat. ॥

49 Une caiche est un voilier à deux mâts.

$50 \mathrm{BnF}$ : Clairambault ms 887, fo63 r०-64 vº lettre autographe de Renau du 12 mars 1694 à la rade de Groye : Essai du Le Bon.

51 Bibliothèque municipale de Bordeaux : ms 733, doc. 24. 


\section{La mise en œuvre de la méthode de Renau d'Élissagaray}

eut toujours une presence d'esprit admirable, il voulut toujours se battre à brûle pourpoint, \& au milieu du feu il donna toujours ses ordres comme auparavant, \& avec la même tranquillité $॥$.

Mais les épreuves du comportement du vaisseau à la mer continuent, avec les modifications apportées pour le perfectionner, puisque le 8 juin Renau écrit :

Le vaisseau Le Bon n'a pas pû sortir de ce port parce qu'il a fait un mauvais temps et vent contraire depuis trois jours [...]. Je ne fais rien changer aux œuvres mortes de ce vaisseau quoiqu'il les ait trop en volume pour un vaisseau fin de voille afin qu'il soit consideré dans son estat le plus desavantageux et que les bonnes qualitez qu' on luy pourra remarquer ne soient attribuées qu'a la bonté de ces fonds et je puis vous assurer Monseigneur que je les jugeray aussy seurement que pourroit faire personne et que je pencheray plus du costé de la defiance que de celuy de la prevention en faveur de ma construction.

On lit, annoté en marge : « on est persuadé qu'il en rendra compte fidelement s'appliquer a en observer les bonnes et mauvaises qualités $\|{ }^{52}$.

Le 19 juin, Pontchartrain peut écrire : « Je suis bien aise que la construction du vaisseau Le Bon ayt reussy. Sa Majesté fera faire suivant cette methode les premiers vaisseaux qu'elle fera bastir $"{ }^{53}$.

Une correspondance de Renau au ministre du jer avril 1695 fait encore état d'un « petit bastiment que je vous ay fait faire ". II " vaudra plus que beaucoup d'autre que le Roy a, avant de prendre aucune resoIution sur cette construction il seroit bon que j'eusse I'honneur de vous entretenir un quart d'heure avec un modele en bois que j'ay fait faire pour pouvoir vous expliquer certaines choses qui seront de consequence $1{ }^{54}$.

Les années qui suivent sont, pour Renau, essentiellement consacrées à des fonctions d'ingénieur du roi détaché à la marine, soit de fortification des côtes. Il est aussi chargé d'organiser deux expéditions destinées entre autres à des relevés de cartes et à projeter des travaux de fortification en 1696 et 1699-1700, la première, à Saint-Domingue et la deuxième à la Guadeloupe.

52 AN : Mar B4 $15 f^{\circ} 168$, lettre de Renau à Pontchartrain. 53 AN : Mar B2 98 f $^{\circ} 638$, lettre de Pontchartrain à Renau du 19 juin 1694.

54 AN : Mar D1 21, lettre de Renau à Pontchartrain du ler avril 1695.
Le 10 mars 1701, Renau qui est sur le point de quitter Paris pour Madrid, profite d'une réponse qu'il doit faire à Pontchartrain sur des réflexions de Langeron concernant la construction navale pour évoquer les idées directrices de la proposition, qu'il avait faite à Seignelay en février 1684, d'une organisation des constructions dont il serait en quelque sorte le grand ordonnateur :

L'important seroit de regler les fonds et de donner sur cela la perfection mais je ne crois pas que l'on y puisse parvenir par des disputes et des contestations semblables à celles que l'on a desja veües pendant plus de dix ou douze ans qui n'ont servy qu' a gaster beaucoup de vaisseaux du Roy. C'est plustost l'ouvrage d'un seul homme que l'opinion de plusieurs et c'est une matière bien profonde et d'une grande experience qui demande au moins autant de mathematiques que d'experience et autant d'experience que de mathematiques ce qui est bien difficile de trouver dans un grand nombre de gens et je crois plus possible d'accorder les jansenistes avec les molinistes, que d'accorder sur cela les sentimens de la marine la pluspart parlant sans idee et attribuant des effets à des causes qui en produiroient d'autres opposées, dont la connoissance depend de beaucoup de physique et de mathematiques dont ils ne sçavent pas seulement les principes, souvent on croit remedier à un inconvenient que l'on tombe dans vingt autres. Enfin Mgr c'est la matiere la plus delicate et la plus composée que les mathematiques puissent avoir pour objet quand on aura regle quelque chose sur cela et que vous me ferez l'honneur de m'en faire part, je prendray la liberté de vous en dire mon sentiment ${ }^{55}$.

On sait que Renau, ingénieur au service de Philippe V durant la Guerre de Succession d'Espagne, ne rentra définitivement en France qu'en mai 1709. On construisit peu de navire durant la période qui suivit et nous n'avons pas retrouvé de documents faisant état d'une activité particulière de Renau dans ce domaine. En revanche, on trouve aux Archives nationales à l'année 1734 le 13 janvier, une lettre de Pierre Bouguer à Maurepas où il est question de Renau. Bouguer commence à réfléchir à son Traité du navire qu'il ne publiera que douze ans plus tard ${ }^{56}$. Son projet qui est de parvenir à dissiper l'incertitude liée aux multiples méthodes des charpentiers s'apparente à celui de

55 AN : Mar D/1/10 (f० 72-73), lettre de Renau au ministre Pontchartrain.

56 Pierre Bouguer, Traité du navire, de sa construction, et de ses mouvements, Paris, Jombert, 1746. 
Renau et dès lors, savoir au juste ce qu' a fait Renau se justifie. D'après la lettre, il semble bien que l'idée d'y aller voir de plus près lui est suggérée par Maurepas auquel il écrit :

Je n'ai point vû ce que feu Mr le Chevalier Renau a fait sur la Construction ; mais je sçai cependant bien que son travail n'a eu pour objet, que de donner aux constructeurs une plus grande facilité de tracer les lignes courbes qu'on fait ordinairement entrer dans les gabaris; de former des plans d'une façon plus régulière et plus commode, et de fournir enfin un certain nombre déterminé de figures qu'on peut donner avec succès aux vaisseaux. C'est là Votre Grandeur le sçait mieux que personne, tout l'usage que Mr le Chevalier Renau a fait de la Géométrie : Mais je suis persuadé qu'on en feroit un autre pour le moins aussi considérable, si l'on éclairoit les pratiques des Constructeurs; et qu'au lieu d'insister sur la maniere de tracer les lignes courbes, on insistât sur la maniere de choisir entre ces lignes, et de découvrir, sans être toujours obligé d'en venir à l'expérience, les propriétés qu'elles ont par raport à la construction.

Il termine sa lettre en demandant à Maurepas de lui « faire communiquer ce que Mr Renau a fait sur cette matiere ॥. Peu après, Maurepas se fait envoyer le procès-verbal de la visite et décision de la construction de la Serpente, bastie suivant les proportions du Sr Renau ${ }^{57}$.

$57 \mathrm{BnF}:$ n.a.f. ms 9483 p 55.
Ainsi, ce que l'on retient, dans les années 1730, de la tentative de Renau, est bien ce que notre examen des archives confirme. La question que nous nous posions - pourquoi le roi accorda-t-il suffisamment de confiance à Renau pour lui permettre de faire construire, dans les années 1690, selon ses propres formes et proportions - semble être fondée en bonne part sur l'expérience qu'il avait pu acquérir pendant les années 1680 lorsqu'il fut chargé de régulariser les tracés des différents charpentiers. Ce travail de réduction des différentes manières en usage, faisait de lui le mieux à même de les comparer et de déterminer les rapports entre leurs caractéristiques et les qualités nautiques des bâtiments construits. Ce dont rend compte son mémoire sur les constructions de février 1684.

Mais on peut penser aussi que les proportions et formes auxquelles il avait abouti et où l'on reconnaît, selon Bouguer, la détermination d'un certain nombre de figures qu'on peut donner au vaisseau, est en particulier le fruit du travail collectif mené par Tourville, Pangalo et Renau à Toulon, durant I'hiver 1685-1686. Soit la collaboration de trois hommes d'exception attachés à combiner au plus juste leurs compétences respectives, celle d'un officier de mer, d'un charpentier et d'un ingénieur, pour choisir entre les figures possibles et tirer le meilleur parti des connaissances existantes. 